Research Paper

\title{
Post-reperfusion Syndrome during Renal Transplantation: A Retrospective Study
}

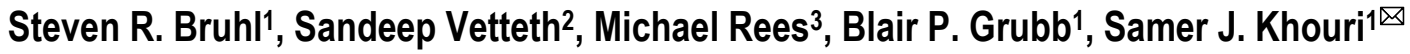 \\ 1. Division of Cardiology, 3000 Arlington Ave., Toledo, OH 43537, USA \\ 2. Division of Nephrology, 3000 Arlington Ave., Toledo, $\mathrm{OH} 43537$, USA \\ 3. Department of Urology, 3000 Arlington Ave., Toledo, OH 43537, USA
}

Corresponding author: Samer Khouri, MD, FACC, FASE. Associate Professor of Medicine. Director, Echocardiography Lab. Division of Cardiovascular Medicine, University of Toledo, Health Science Campus, Mail Stop 1118, Toledo, Ohio 43614. Tel 419-383-3697. Fax 419-383-3041. Samer.khouri@utoledo.edu

(C) Ivyspring International Publisher. This is an open-access article distributed under the terms of the Creative Commons License (http://creativecommons.org/ licenses/by-nc-nd/3.0/). Reproduction is permitted for personal, noncommercial use, provided that the article is in whole, unmodified, and properly cited.

Received: 2012.04.13; Accepted: 2012.07.09; Published: 2012.07.16

\begin{abstract}
Post-reperfusion syndrome (PRS) is a serious, widely reported complication following the reperfusion of an ischemic tissue or organ. We sought to determine the prevalence, risk factors and short-term outcomes of PRS related renal transplantation. We conducted a retrospective, case-control study of patients undergoing renal transplantation between July 2006 and March 2008. Identification of PRS was based on a drop in mean arterial pressure by at least $15 \%$ within 5 minutes of donor kidney reperfusion. Of the 150 consecutive renal transplantations reviewed, 6 patients (4\%) met criteria for post-reperfusion syndrome. Univariate analysis showed that an age over 60, diabetes mellitus, Asian race, and extended criteria donors increased the odds of developing PRS by 4.8 times ( $95 \% \mathrm{Cl}[1.2,20] ; P=.0338$ ), 4.5 times (95\% Cl [I.II, I8.8]; $P=.0378)$, 35.5 times (95\% Cl [3.94, 319.8]; $P=0.0078$ ) and 9.6 times $(95 \% \mathrm{Cl}[1.19,76.28] P=0.0 \mathrm{II})$ respectively. Short term follow-up revealed increased graft failure rate within 6 months $(6 \%$ vs. $16 \% P=0.0125)$ and almost twice the number of hospital days post-transplant in PRS cohorts $(5.43 \pm 2.29$ vs. $10.8 \pm 7.29 P=<0.000 I)$. Despite limited reporting, PRS appears to be a relatively common complication of renal transplantation and is associated with increase morbidity.
\end{abstract}

Key words: Post reperfusion syndrome, PRS, renal transplant, transplant complications, hypotension.

\section{Introduction}

In 1987 Aggarwal described post-reperfusion syndrome (PRS) as the sudden development of hypotension occurring within 5 minutes of liver reperfusion, lasting at least 1 minute in duration, and commonly associated with persistent bradycardia [1]. A recent study of PRS during liver transplantation showed that patients who developed PRS required longer mechanical ventilation times, longer intensive care unit stays, and were 2.5 times more likely to require re-transplantation than those without PRS.[2]
However, despite over three decades of research, PRS is still a common surgical complication occurring in approximately $20-30 \%$ of all liver transplants.[3, 4] More recently, PRS has also been reported during several other surgeries including cardiopulmonary bypass, ischemic limb reperfusion, aneurysm repair, and most recently renal transplantation at largely unknown frequencies.[5-7]

Although the exact etiology of PRS remains unknown, PRS should not be confused with ischem- 
ic-reperfusion injury, which is a phenomenon largely isolated to the ischemic organ itself and occurs over days. In contrast, PRS is a systemic phenomenon that by definition occurs within 5 minute of organ reperfusion and has been shown to adversely affect not only the re-perfused organ, but also have profound effects on the autonomic and cardiovascular systems.[3] Give the chronological proximity of the syndrome to reperfusion, initial theories about the etiology of PRS centered on the hypothesis that a sudden relative hyperkalemia, acidosis, and/or hypothermia develops at reperfusion due to a sudden shift of cold preservation solution, potassium, lactate, or calcium into systemic circulation from the transplanted organ. However, this theory was significantly weakened by a prospective study showing that the only statistically significant correlation between a patient's core temperature, potassium levels, calcium levels, arterial blood-gas tensions, serum $\mathrm{pH}$, hemodynamic parameters, or PRS was a decrease in systemic vascular resistance (SVR). This lack of correlation between the studied variables and PRS suggests that the agent or agents responsible for the systemic vasodilatation remained unknown.[3] Another study showed that the cardiac preload in PRS patients was significantly decreased as compared to non-PRS cohorts despite equivalent left ventricular ejection fractions on trans esophageal echocardiography.[8] Finally, increased levels of neutrophil and macrophage activation with concomitant anaphylatoxin formation has been identified in patients experiencing PRS compared to controls suggesting that an immunologic cause may be at least partially responsible for the syndrome.[9]

Extensive research has also been done to prevent PRS from occurring including the use of alternative preservation solutions,[10] transplant flushing just prior to reperfusion,[11] various alterations in the reperfusion sequences and rates, $[5,12]$ as well as pre-treatment of patients with various drugs.[13] Although the results these interventions have been mixed, the overall prevalence of PRS during liver transplantation has remained relatively constant.[10]

Regardless of the cause, the effects of PRS are clearly detrimental. The development of PRS during liver transplantation has been shown to be a significant risk factor for poor initial transplant function and increased mortality ( $80 \%$ vs. $96 \%$; $\mathrm{P}=0.04)$ making the risk of death 2.87 times greater than non-PRS cohorts $([1.41,5.85] ; P=0.04) .[3,4]$ The same study also showed an increased prevalence of severe post-operative renal failure ( $70 \%$ vs. $36 \% ; \mathrm{P}=0.03$ ) with a relative risk 3.3 times that of patients without PRS $([1.7,6.4] ; P=0.01)$.
With the recent report of PRS during renal transplant,[6] the primary goal of this study was to establish the prevalence, risk factors, and potential short term complications associated with PRS during renal transplantation.

\section{Materials and Methods}

After Institutional Review Board approval, data was retrospectively collected on 150 consecutive patients undergoing renal transplantation in an academic hospital.

Identification of PRS was based on a drop in mean arterial pressure (MAP) of at least $15 \%$ within 5 minutes of donor kidney reperfusion and lasting at least 1 minute. Baseline recipient variables collected included age, gender, race, left ventricular ejection fraction (LVEF), deceased donor vs. living donor transplantation, race, creatinine at time of transplant, hypertension, diabetes mellitus, a history of coronary artery disease, beta blocker or calcium channel blocker use within 24 hours, preoperative potassium levels and hemoglobin levels. Donor variables collected included race, terminal creatinine, and the use of machine perfusion preservation of the donor organ prior to transplantation. In addition, the quality of donor organs were classified into one of three United Network for Organ Sharing (UNOS) defined categories including donors after cardiac death (DCD) who are donors who have suffered devastating and irreversible brain injury that may be near death, but did not meet formal brain death criteria. Second, expanded criteria donors (ECD) were defined as donors over age 50 that have high blood pressure, a creatinine above $1.5 \mathrm{mg} / \mathrm{dl}$ and/or the donor passed away from a CVA. Finally standard criteria donors (SCD) were defined as donors who did not meet DCD or ECD criteria. Follow up statistics included delayed graft function, graft failure within 6 months and 1 year, mortality, and hospital days post-transplant.

Preoperatively, all patients underwent immunosuppressive induction with a combination of alemtuzumab 30mg and mycophenolate $720 \mathrm{mg}$, as well as premedication with $25 \mathrm{mg}$ of diphenhydramine, $500 \mathrm{mg}$ of methylprednisolone, $650 \mathrm{mg}$ of acetaminophen and $20 \mathrm{mg}$ of famotidine. Just prior to restoration of graft reperfusion by renal arterial clamp removal, all patients were also given furosemide 40 $\mathrm{mg}$, mannitol $12.5 \mathrm{~g}$ and $500 \mathrm{mg}$ of methylprednisolone. Only two surgeons performed all of the renal transplants under study.

\section{Statistical Method}

All study variables were analyzed descriptively between the two cohorts (PRS vs. no PRS). Univariate 
analyses of patient characteristics between the two outcome cohorts were also conducted. Numbers and percent's were provided for dichotomous and polychotomous variables and means and standard deviations were provided for continuous variables. A Student's $t$ test was used to compare all continuous variables and a chi-square test or Fischer's exact test was used for all categorical variables. Univariate analysis was used to estimate odds ratios and the associated 95\% confidence intervals (CI). Multiple regression analysis was also performed on all statistically significant variables in order to determine independence. A two-sided $P$ value of less than 0.05 was considered statistically significant. All analysis was performed using Graph Pad Prism version 5.0 (La Jolla, CA) and SAS.

\section{Results}

Of the 150 consecutive renal transplantations reviewed between July 2006 to March 2008, 6 patients (4\%) were diagnosed as having developed post-reperfusion syndrome. Table 1 describes the baseline patient demographics and transplant characteristics of the two cohorts. Compared to patients without PRS, the PRS cohorts had a statistically significant increase in the prevalence of patients over 60 years old $(66.67 \%$ vs. $27.08 \%$; $P=0.0358)$, diabetes mellitus (83.33\% vs. $32.64 \% ; P=0.0153)$, Asian race (33\% vs. $1 \%$; $(\mathrm{P}=<0.0001)$ and transplantation from expanded criteria donors ( $9 \% \mathrm{vs}$. $50 \% P=0.0176$ ). Similarly, patients with a

Table I. Baseline characteristics of patients with and without PRS history of coronary artery disease $(32.6 \%$ vs. $66.67 \%$ $P=0.0858$ ), and patients receiving a kidney from self-described black donors ( $10 \%$ vs. $33 \% P=0.0671)$ also showed a strong trend toward statistical significance as risk factors. Conversely, patients receiving SCD transplants as opposed to transplants from ECD or DCD had almost $1 / 10^{\text {th }}$ the prevalence of PRS ( 2 of 87 vs. 2 of 10), while the prevalence of PRS in white patients ( $70 \%$ vs. $33 \% \mathrm{P}=0.0574$ ) as well the prevalence when both donor and recipient were white $(68 \%$ vs. $33 \% \mathrm{P}=0.0754$ ) was markedly less and showed strong trends toward statistical significance as protective factors.

\begin{tabular}{|c|c|c|c|}
\hline Characteristics & No PRS $(N=144)$ & $\operatorname{PRS}(\mathrm{N}=0)$ & $P$ \\
\hline Recipient Age,y & $50.53 \pm 14.4$ & $61.5 \pm 5.3$ & 0.0654 \\
\hline Age $>60$ & $39(27)$ & $4(67)$ & 0.0358 \\
\hline Donor Age & $39.9 \pm 13.8$ & $38 \pm 16.6$ & 0.7490 \\
\hline Patient BMI & $28.4 \pm 5.6$ & $294 \pm 4$ & 0.6027 \\
\hline Male & $90(63)$ & $3(50)$ & 0.5397 \\
\hline \multicolumn{4}{|l|}{ Donor by race } \\
\hline White & $125(87)$ & $4(67)$ & 0.1658 \\
\hline Black & $14(10)$ & $2(33)$ & 0.0671 \\
\hline Hispanic & $5(3)$ & 0 & \\
\hline \multicolumn{4}{|l|}{ Recipient by race } \\
\hline White & $101(70)$ & $2(33)$ & 0.0574 \\
\hline Black & $32(22)$ & $1(17)$ & 0.7496 \\
\hline Hispanic & 9 (ด) & $1(17)$ & 0.3195 \\
\hline Asian & $2(1)$ & $2(33)$ & $<0.0001$ \\
\hline \multicolumn{4}{|l|}{ Race of donor and recipient } \\
\hline White to white & $97(68)$ & $2(33)$ & 0.0754 \\
\hline White to non-white & $27(19)$ & $2(33)$ & 0.3789 \\
\hline Non-white to white & $3(2)$ & 0 & \\
\hline Non-white to non-white & $16(11)$ & $2(33)$ & $0 . \overline{10} 21$ \\
\hline \multicolumn{4}{|l|}{ Recipient Medical history } \\
\hline Hypertension & $135(94)$ & $6(100)$ & \\
\hline Diabetes mellitus & $50(33)$ & $5(83)$ & $0 . \overline{0153}$ \\
\hline Coronary artery disease & $46(33)$ & $4(67)$ & 0.0858 \\
\hline \multicolumn{4}{|l|}{ Baseline therapies } \\
\hline Beta blockers & $87(61)$ & $4(67)$ & 0.7607 \\
\hline Calcium channel blockers & $59(44)$ & $2(33)$ & 0.7112 \\
\hline \multicolumn{4}{|l|}{ Baseline lab values } \\
\hline Hemoglobin ${ }^{2}$ & $12.3 \pm 1.6$ & $11.9 \pm 0.9$ & 0.6002 \\
\hline Senum potassium, Meq/ $\mathrm{L}^{\mathrm{b}}$ & $4.4 \pm 0.7$ & $4.4 \pm 0.5$ & 0.9297 \\
\hline LVEF $^{c}$ & $54 \pm 9.8$ & $61.5 \pm 5.3$ & 0.3396 \\
\hline \multicolumn{4}{|l|}{ Donor criteria } \\
\hline Living & $49(34)$ & $2(33)$ & 0.9722 \\
\hline Deceased & $95(60)$ & $4(67)$ & 0.9722 \\
\hline Standard & $85(90)$ & $2(50)$ & 0.0176 \\
\hline Extended & $9(9)$ & $2(50)$ & 0.0112 \\
\hline After cardiac death & $1(1)$ & 0 & \\
\hline Deceased donor ischemic time, hours & $14.1 \pm 4.8$ & $8.5 \pm 0.7$ & 0.0934 \\
\hline Live donor ischemic time, hours ${ }^{e}$ & $3 \pm 0.6$ & NA & \\
\hline Pumped by OPO or TXC & $22(15)$ & $1(10)$ & $0 . \overline{25} 65$ \\
\hline $\begin{array}{l}\text { Continuous variables are shown as mean values } \\
\text { of patients. PRS indicates post reperfusion syndr } \\
\text { procurement organization; TXC, transplant center } \\
\text {-No PRS has } 2 \text { patients with missing data. FNo PR } \\
\text { 'No PRS has } 5 \text { patients with missing data. } \\
\text {-61 No PRS patients had no LVEF measured or av } \\
\text { 'No PRS has } 65 \text { patients and PRS has } 4 \text { patients } \\
\text {-No PRS has } 116 \text { patients with missing data, PRS }\end{array}$ & $\begin{array}{l}\text { standard deviations and } \\
\text { ome; LVEF, left ventricul } \\
\text { NA indicates not availa } \\
\text { Shas } 5 \text { patients with mi } \\
\text { ailable. }\end{array}$ & $\begin{array}{l}\text { ary variables are } \\
\text { ection fraction. } 0 \\
\text { data. }\end{array}$ & $\begin{array}{l}\text { own as n (\%) } \\
0, \text { organ }\end{array}$ \\
\hline
\end{tabular}


Univariate analysis showed that an age over 60 years increased the odds of developing PRS by 4.8 times $(95 \% \mathrm{CI}[1.2,20] P=0.0338)$, diabetes mellitus by 4.47 times (95\% CI $[1.11,18.08] P=0.0378)$, Asian race by 35.5 times $(95 \% \mathrm{CI}[3.94,319.8] \mathrm{P}=<0.01)$ and extended criteria donor by 6.7 times (95\% CI [1.091, 41.16] $\mathrm{P}=0.0737$ ) that of standard criteria donors (Table 2). Multivariate regression analysis including age over 60 years, diabetes mellitus, Asian race and extended criteria donor showed diabetes mellitus $(\mathrm{P}=0.043)$ and Asian race $(\mathrm{P}<0.001)$ were independent risk factors for the development of PRS.

Short term follow up results of the study subjects revealed an increased risk of graft failure within 6 months ( $6 \%$ vs. $16 \% P=0.0125)$ and almost twice the number of hospital days post-transplant in the PRS cohort (5.43 \pm 2.29 vs. $10.8 \pm 7.29 P=<0.0001)$ (Table 3$)$.

\section{Discussion}

Although post-reperfusion syndrome has been extensively studied in liver transplantation, this is the first study to investigate the prevalence of PRS during renal transplantation.

Part of the reason for the paucity of renal transplant related PRS studies in the literature may be related to its decreased prevalence compared to liver transplantation. While the prevalence of PRS in liver transplantation remains between $20-30 \%$, this study suggests that PRS associated renal transplantation is closer to $4 \%$.

Table 2. Univariate analysis of variables associated with PRS

\begin{tabular}{|c|c|c|c|c|}
\hline \multirow[b]{2}{*}{ Characteristics } & \multicolumn{3}{|c|}{$95 \%$ Confidence Interval } & \multirow[b]{2}{*}{$P$} \\
\hline & Odds & Lower & Upper & \\
\hline Age $>60$ & 4.80 & 1.20 & 20.00 & 0.0338 \\
\hline Male & 0.60 & 0.12 & 3.08 & 0.6741 \\
\hline \multicolumn{5}{|l|}{ Recipient by race } \\
\hline White & 0.23 & 0.04 & 1.33 & 0.0957 \\
\hline Asian & 35.50 & 3.94 & 319.80 & $<0.01$ \\
\hline \multicolumn{5}{|l|}{ Donor by race } \\
\hline Black & 1.37 & 0.15 & 12.18 & 1.0000 \\
\hline \multicolumn{5}{|l|}{ Donor Criteria } \\
\hline Standard & 0.11 & 0.01 & 0.85 & 0.0121 \\
\hline Extended & 9.56 & 1.20 & 76.28 & 0.0115 \\
\hline After cardiac death & 1.27 & 0.23 & 7.18 & 1.0000 \\
\hline \multicolumn{5}{|l|}{ Medical history } \\
\hline Hypertension & 0.91 & 0.05 & 17.44 & 1.0000 \\
\hline Diabetes mellitus & 4.47 & 1.11 & 18.08 & 0.0378 \\
\hline Coronary artery disease & 3.17 & 0.85 & 11.79 & 0.0897 \\
\hline \multicolumn{5}{|l|}{ Baseline therapies } \\
\hline Beta blockers & 1.30 & 0.23 & 7.40 & 1.0000 \\
\hline Calcium channel blockers & 0.72 & 0.13 & 4.06 & 1.0000 \\
\hline
\end{tabular}

PRS, post reperfusion syndrome

Table 3. Short term follow up characteristics of patients with and without PRS

\begin{tabular}{lccc} 
Characteristics & No PRS $(\mathrm{N}=144)$ & PRS (N=6) & $P$ \\
\hline Delayed graft function & $16(11)$ & $1(16)$ & 0.68 \\
Graft failure within 6 months & $9(6)$ & $1(16)$ & 0.01 \\
Graft failure within 1 year & $20(14)$ & $1(16)$ & 0.85 \\
Mortality within 1 year & $8(5)$ & $0.00 \%$ & NS \\
Hospital days post transplant & $5.433 \pm 2.29$ & $10.8 \pm 7.27$ & $<0.0001$ \\
\hline
\end{tabular}

Continuous variables are shown as mean values \pm standard deviations and binary variables are shown as $\mathrm{n}$

(\%) of patients. PRS indicates post reperfusion syndrome. 
A second reason for decreased reporting may be due to the less severe hypotension seen during renal transplantation. During liver transplantation, any drop in MAP less than $30 \%$ is considered only mild PRS. In our study, the average drop in MAP was only $24 \%$, and only 1 of the 6 patients had a drop $>30 \%$. The reason we chose a drop in MAP of $>15 \%$ as the cutoff relates to the proposed mechanism of PRS itself. Since the prevailing theory regarding the cause of PRS centers around an unknown agent or agents being released from the transplanted organ at reperfusion, it stands to reason that a kidney, which weighs less than $1 / 10^{\text {th }}$ that of a transplanted liver, would contain less of this substance.[14] However, one should not assume that the less dramatic hypotension of renal transplant PRS automatically correlates with less severe morbidity. Although hypotension lasting greater than 1 minute might be expected to cause some degree of adverse consequences, most research to date suggest that the post-reperfusion hypotension is simply a marker of a more serious underlying immunological, inflammatory or other unknown process occurring at a molecular level.[3, 10] The fact that this study also showed increased rates of 6 month graft failure and increased post-operative hospital stays in PRS cohorts also seems to support this hypothesis.

This study is also the first to suggest that advanced age, Asian race, extended criteria donor kidneys, and diabetes mellitus might be risk factors for the development of PRS. One plausible explanation for diabetes as a risk factor may be found in a separate study showing that patients diagnosed with autonomic dysfunction had an increased prevalence of PRS as compared to those without autonomic dysfunction. Although that study performed rigorous testing to diagnose autonomic dysfunction prior to transplantation, the presence of diabetes, especially longstanding, uncontrolled diabetes severe enough to cause renal failure may be a surrogate marker for predicting autonomic dysfunction and subsequent PRS in surgical patients.[15]

Interestingly, not only was an ECD associated with an almost 10 fold increase in the risk for developing PRS, but a recipient's age over 60 years was also associated with an increased risk of developing PRS. Since one of the criteria for ECD's is an age $\geq 60$, this would seem to suggest that some characteristic of advanced age might explain both risk factors.

The fact that Asian race appeared to be an independent risk factor for the development of PRS was one of the most unexpected findings of the study since Asian race has never previously been reported as a risk factor. Although it is certainly possible that this finding is completely coincidental, the fact that trans- plant complications related to race are already well known to transplant medicine suggests that this finding warrants further investigation. Furthermore, if this variable is validated as a true risk factor for PRS, it may give credence to the hypothesis that PRS is caused by an immunological reaction that perhaps is more likely to occur in Asian recipients.

Although coronary artery disease showed only a strong trend toward statistical significance, we believe that this may have reached statistical significance in a larger patient population. Aggarwal et al. showed that patients experiencing PRS had a significantly lower cardiac output 30 seconds after reperfusion despite concomitant decreases in SVR and mean arterial pressure. Although the reason for this is unclear, a history of CAD may be a marker of patients with decreased cardiac reserve due to significant coronary artery stenosis. Generally speaking, hypotension leads to a systemic vasconstrictive response and a concomitant attempt at increasing cardiac output by increasing heart rate and/or contractility. If systemic vasoconstriction fails, as in PRS, pre-existing coronary stenosis may attenuate the hearts ability to increase coronary blood flow enough to meet the new cardiac demands. Since bradycardia is relatively common in classic PRS, increased contractility and coronary blood flow become even more essential to meeting cardiac demand.[2]

\section{Limitations}

One of the largest limitations of this study is its relatively small sample size and retrospective design. Not only are odds ratios less powerful than risk ratios, but the collection of data such as a numerical representations of LVEF and cold ischemic times were limited by sporadic documentation as is typical of retrospective studies. Our cutoff for a drop in MAP being $>15 \%$ is also a potential criticism. Although we considered using both higher and lower cutoffs, ultimately we felt that a cutoff higher than $15 \mathrm{mmHg}$ would decrease the sensitivity and introduce false negatives due to the significantly size difference between a kidney and liver transplant, while a lower cutoff would potentially introduce false positives due to normal background variation in blood pressure. A final limitation is that although renal transplant related PRS likely occurs by a similar mechanism to that of liver transplantation, a prospective rather than retrospective study investigating SVR and cardiac output is needed in order to confirm or refute this hypothesis.

\section{Conclusion}

Despite limited reporting, post reperfusion syn- 
drome appears to be a relatively common complication of renal transplantation. Patients with a history of diabetes mellitus, age greater than 60 , and the use of extended criteria organs appear to be at increased risk of developing PRS during renal transplantation. Although it is currently unclear whether these findings can be extrapolated to other PRS associated surgeries; the increased rates of kidney failure at 6 months and post-transplant hospital stays nearly double that of non-PRS cohorts strongly support increase morbidity when PRS develops in renal transplantation. Finally, although these findings are thought provoking, a prospective controlled trial is required to further validate these findings and to more fully determine the clinical significance of developing PRS during renal transplantation.

\section{Competing Interests}

The authors have declared that no competing interest exists.

\section{References}

1. Aggarwal $\mathrm{S}$, et al. Postreperfusion syndrome: cardiovascular collapse following hepatic reperfusion during liver transplantation. Transplantation proceedings, 1987. 19: 54-5.

2. Hilmi I, et al. The impact of postreperfusion syndrome on short-term patient and liver allograft outcome in patients undergoing orthotopic liver transplantation. Liver Transpl, 2008. 14(4): 504-8.

3. Aggarwal S, et al. Postreperfusion syndrome: hypotension after reperfusion of the transplanted liver. J Crit Care, 1993. 8(3): 154-60.

4. Paugam-Burtz C, et al. Postreperfusion syndrome during liver transplantation for cirrhosis: outcome and predictors. Liver Transpl, 2009. 15(5): 522-9.

5. Beyersdorf $\mathrm{F}$ and Schlensak $\mathrm{C}$. Controlled reperfusion after acute and persistent limb ischemia. Semin Vasc Surg, 2009. 22(1): 52-7.

6. Bruhl S.R, et al. Postreperfusion syndrome during living-related donor renal transplantation: a case report. Transplant Proc, 2008. 40(5): 1754-5.

7. Kodakat S.K, et al. A case of post-reperfusion syndrome following surgery for liver trauma. Br J Anaesth, 2006. 96(1): 31-5.

8. de la Morena $G$, et al. Ventricular function during liver reperfusion in hepatic transplantation. A transesophageal echocardiographic study. Transplantation, 1994. 58(3): 306-10.

9. Tomasdottir H, Bengtson J.P, and Bengtsson A. Neutrophil and macrophage activation and anaphylatoxin formation in orthotopic liver transplantation without the use of veno-venous bypass. Acta Anaesthesiol Scand, 1996. 40(2): 250-5.

10. Ko J.S, et al. Greater hemodynamic instability with histidine-tryptophan-ketoglutarate solution than University of Wisconsin solution during the reperfusion period in living donor liver transplantation. Transplant Proc, 2008. 40(10): 3308-10.

11. Homvises B, et al. The minimal flush volume for washout of preservation fluid in liver transplantation. Transplant Proc, 2008. 40(7): 2123-6.

12. Daniela $\mathrm{K}$, et al. Influence of retrograde flushing via the caval vein on the post-reperfusion syndrome in liver transplantation. Clin Transplant, 2004. 18(6): 638-41.

13. Acosta F, et al. Atropine prophylaxis of the postreperfusion syndrome in liver transplantation. Transplant Proc, 1999. 31(6): 2377.

14. Cotran R.S.K, et al. Robbins and Cotran pathologic basis of disease. St Louis, MO: Elsevier Saunders. 2005:878.

15. Perez-Pena J, et al. Autonomic neuropathy is associated with hemodynamic instability during human liver transplantation. Transplant Proc, 2003. 35(5): 1866-8. 